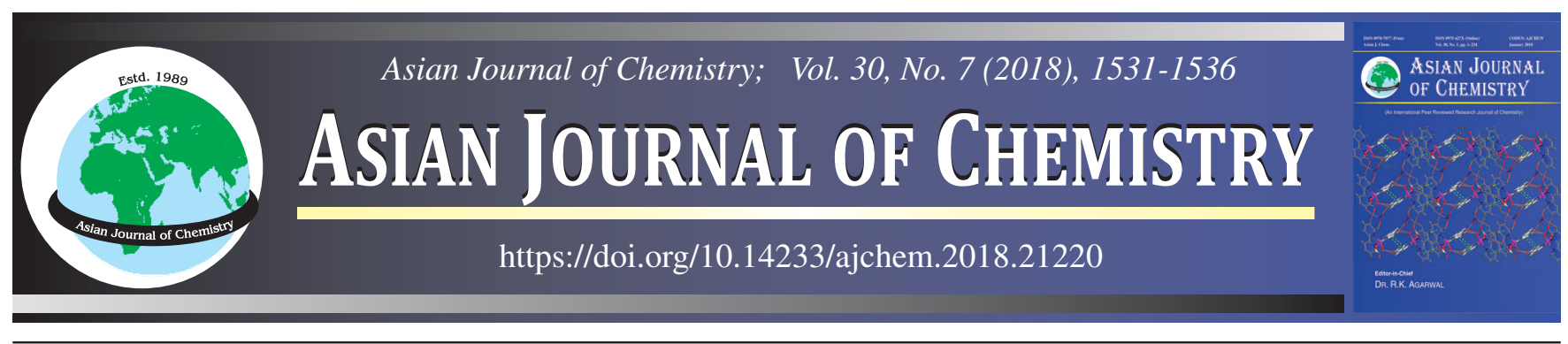

\title{
Green Synthesis, Characterization and Antidiabetic Activity of 2-Substituted Aryl/Alkyl-N-Aryl/Alkyl Imidazo[1,2-a]pyridin-3-amine Derivatives
}

\author{
Tata Veereswara Rao Kota ${ }^{1,2, *}$, Hima Bindu Gandham ${ }^{3}$ and Paul Douglas Sanasi ${ }^{3}$
}

${ }^{1}$ Department of Chemistry, Bonam Venkata Chalamayya Engineering College, Odalarevu, Allavaram Mandal-533 201, India

${ }^{2}$ Department of Chemistry, Jawaharlal Nehru Technological University Kakinada, Kakinada-533 003, India

${ }^{3}$ Department of Engineering Chemistry, AU College of Engineering (A), Andhra University, Visakhapatnam-530 003, India

*Corresponding author: E-mail: veresh.kota@gmail.com

Received: 10 January 2018;

Accepted: 23 February 2018;

Published online: 31 May 2018;

AJC-18926

Imidazo[1,2- $a$ ]pyridines containing ring junction have shown broad therapeutic properties in the drug discovery sector, such as the treatment of heart disease, migraines, digestive disease and viral disease. Imidazo[1,2-a]pyridine structure is very much prevalent in several marketed drugs viz., olprinone, zolpidem, levamisole, alpidem, zolimidine etc. The present paper describes the green synthesis of some novel imidazo[1,2-a]pyridine derivatives (6a-f/7a-f), a straight forward, isocyanide based Groebke-Blackburn-Bienayme, three component reactions in which aldehyde, 2-aminopyridine and isocyanide are condensed in presence of green parameters such as (i) $\mathrm{ZnO}$ nanoparticles $/ \mathrm{ZnFe}_{2} \mathrm{O}_{4}$ nanoparticles (reusable heterogeneous catalysts), (ii) 2-methyl-tetrahydrofuran (green solvent) and (iii) microwave irradiation (energy efficiency reducing time period of the reaction). These derivatives were evaluated for antidiabetic experiments and the results revealed that most of the compounds were active towards antidiabetic property.

Keywords: Green synthesis, Imidazo[1,2-a]pyridine, Antidiabetic, 2-Amino-pyridine, $\mathrm{ZnO}$ nanoparticles, $\mathrm{ZnFe}_{2} \mathrm{O}_{4}$ nanoparticles.

ᄂ _ - - - - - - - - - - - - - - - - - - - - - - - - - -

\section{INTRODUCTION}

Among the nitrogen derivatives, imidazo-fused heterocycles are becoming more important in medicinal and organic chemistry, as they exhibit a broad spectrum of pharmacological and biological activities, such as antiviral, antibacterial, antifungicidal and anti-inflammatory properties [1]. The significance of imidazopyridine scaffolds in the drug discovery sector is well appreciated because of the appealing benefits they offer. Imidazole fused polyheterocycles containing ring junction nitrogen have attracted considerable interest in medicinal chemistry in view of their uses as anti-inflammatory [2], anticancer [3], antibacterial [4] and antituberculosis [5] agents. The importance of imidazo[1,2-a]pyridine is evident from the fact that it is prevalent in several marketed drugs such as olprinone (cardio tonic agent, a phosphodiesterase-PDE 3 inhibitor) [6,7], zolpidem (hypnotic) [8], levamisole (anticancer) [9,10], alpidem (a nonsedative anxiolytic) [11-13], zolimidine (anti-inflammatory) [14,15]. They are also used in molecular recognition and bio-imaging probes due to their structural characteristics [16-18], sedative agents (saripidem \& necopidem) $[19,20]$. Functionalized heterocyclic scaffolds derived from imidazo[1,2-a]pyridines have shown broad therapeutic properties such as treatment of heart disease
[4], migraines [21], digestive disease [22,23] and viral disease [24].

In this paper, we report the synthesis of some novel imidazo[1,2-a]pyridine derivatives, a straight forward, isocyanide based Groebke-Blackburn-Bienayme [25-27], three component reactions in which aldehyde, 2-aminopyridine and isocyanide are condensed in presence of (i) $\mathrm{ZnO}$ nanoparticles/ $\mathrm{ZnFe}_{2} \mathrm{O}_{4}$ nanoparticles (reusable heterogeneous catalysts), (ii) 2-methyltetrahydrofuran (green solvent), (iii) under microwave irradiation (energy efficiency reducing time period of the reaction). Furthermore, these derivatives were further evaluated for their antidiabetic studies.

\section{EXPERIMENTAL}

All the chemicals were commercially procured from Alfa Aesar. Microwave assisted experiments were conducted using a CEM discovery system in a closed vessel under magnetic stirring. $5 \mathrm{~mL}$ reaction vial, microwave tube: $10 \mathrm{~mL}$ pressure vessel, pyrex were used. Melting points were determined in open glass capillaries on a Stuart SMP30 apparatus and are uncorrected. FT-IR spectra were recorded as $\mathrm{KBr}$ pellets on a Shimadzu FTIR 8400S spectrophotometer. ${ }^{1} \mathrm{H}$ NMR (400 $\mathrm{MHz}$ ) spectra were recorded on a Bruker DPX 400 spectrophoto- 
meter using tetramethylsilane (TMS) as internal standard, $\mathrm{CDCl}_{3}$ and DMSO- $d_{6}$ as solvents. HRMS spectra were recorded on a Xevo QT of mass spectrometer. Silica gel 60 F24 of Merck pre-coated plates were employed for their thin layer chromatography (TLC) analysis to check the purity of the compounds, the spot being located under UV light and iodine vapours.

Synthesis of $\mathrm{ZnO}$ nanoparticles: $2.5 \mathrm{~g}$ of Zinc nitrate $\left[\mathrm{Zn}\left(\mathrm{NO}_{3}\right)_{2} \cdot 4 \mathrm{H}_{2} \mathrm{O}\right]$ was dissolved in $300 \mathrm{~mL}$ distilled water. The $\mathrm{pH}$ of the solution was brought to 8.0 by drop wise addition of ammonium hydroxide solution ( $20 \%$ ). The reaction mixture was then stirred for $1 \mathrm{~h}$ at room temperature and transferred to a Teflon lined steel autoclave and maintained at 100 to $120^{\circ} \mathrm{C}$ for different time periods ranging from 6 to $24 \mathrm{~h}$. The autoclave was then allowed to cool to room temperature. The product was then washed several times with distilled water, filtered and dried at $80{ }^{\circ} \mathrm{C}$ overnight. The reaction can be scaled up without any change in phase and morphology of the samples.

Synthesis of $\mathrm{ZnFe}_{2} \mathbf{O}_{4}$ nanoparticles: Ferric nitrate $(4.87 \mathrm{~g}$ ), zinc nitrate $(3.75 \mathrm{~g})$ and sodium hydroxide $(3.28 \mathrm{~g})$ were mixed together and dissolved in $40 \mathrm{~mL}$ of distilled water. Then $2 \mathrm{~mL}$ of polyethylene glycol (PEG) was added dropwise in the solution under constant stirring at room temperature and the $\mathrm{pH}$ level was maintained around 11 . The mixture was continuously stirred for $0.5 \mathrm{~h}$ and transferred to $50 \mathrm{~mL}$ of Teflon lined autoclave. The autoclave was sealed and maintained at $165^{\circ} \mathrm{C}$ for $16 \mathrm{~h}$ in the furnace and then allowed to reach the room temperature. Finally, the brown precipitated solution was washed several times with distilled water and absolute ethanol. Then the brown precipitates were collected and dried at $60{ }^{\circ} \mathrm{C}$ for $6 \mathrm{~h}$ in hot air oven and characterized.

Synthesis of 2-substituted aryl/alkyl-N-aryl/alkyl imidazo[1,2-a]pyridin-3-amine derivatives (6a-f/7a-f) using $\mathrm{ZnO}$ nanoparticles $/ \mathrm{ZnFe}_{2} \mathrm{O}_{4}$ nanoparticles: To a microwave tube vessel containing 2-methyltetrahydrofuran $(1 \mathrm{~mL})$ and ethanol (catalytic quantity), was sequentially added 2-aminopyridine $(50 \mathrm{mg}, 0.53 \mathrm{mmol})$, corresponding aryl-alkyl aldehydes $4 \mathbf{a}-\mathbf{f}(0.53 \mathrm{mmol}), \mathrm{ZnO}-\mathrm{NPs} / \mathrm{ZnFe}_{2} \mathrm{O}_{4} \mathrm{NPs}$ (10 mol $\%)$ and phenyliso-cyanide $(50 \mathrm{mg}, 0.53 \mathrm{mmol}$ ) and then screwed tightly and heated at $120^{\circ} \mathrm{C}$ by microwave irradiation for 2-5 min. The completion of the reaction was judged by the TLC profile, after cooling, the reaction mixture is taken into the centrifuge tube and subjected to centrifugation, the catalyst sediments settle down at the bottom of the centrifuge tube and for $\mathrm{ZnFe}_{2} \mathrm{O}_{4}$ catalyst is separated magnetically by placing a strong neodymium magnet at the bottom of the microwave dish, after which the solution containing the product was taken off with a pipette. Diluted with 2-methyltetrahydrofuran (10 $\mathrm{mL}$ ), washed with $10 \%$ sodium bicarbonate followed by water $(2 \times 5 \mathrm{~mL})$, brine solution $(5 \mathrm{~mL})$ and the two layers were separated. The aqueous layer was further washed twice with 2-methyl- terahydrofuran $(2 \times 3 \mathrm{~mL})$ and the combined organic phases concentrated in vacuum. The product was further purified with the help of recrystallization using isopropylacetate and $n$-heptane to obtain the desired 2-substituted aryl/ alkyl-N-phenylimidazo-[1,2-a]pyridin-3-amine derivatives (6a-f) in high purity with $80-85 \%$ yield. The catalyst is washed thrice with ethyl acetate, dried and the fresh reactants were introduced into the microwave dish, followed by microwave irradiation allowing the reaction to proceed for the next run.
N,2-Diphenylimidazo[1,2-a]pyridin-3-amine (6a) [28,29]: Pale yellow solid; Yield: $84 \%$; m.p.: $239-240{ }^{\circ} \mathrm{C}$; IR (KBr, $\left.\nu_{\max }, \mathrm{cm}^{-1}\right): 3295$ (-N-H str), 2919 (-C-H str), 1600 (-C=N str), 1437 (-C=C- str), 1182 (-C-N str); ${ }^{1} \mathrm{H} \mathrm{NMR}\left(400 \mathrm{MHz}, \mathrm{CDCl}_{3}\right)$ : $\delta 5.57(\mathrm{~s}, 1 \mathrm{H}), 6.62(\mathrm{~d}, J=8.4 \mathrm{~Hz}, 2 \mathrm{H}), 6.78(\mathrm{t}, J=6.4 \mathrm{~Hz}$, $1 \mathrm{H}), 6.88(\mathrm{t}, J=7.6 \mathrm{~Hz}, 1 \mathrm{H}), 7.26-7.20(\mathrm{~m}, 3 \mathrm{H}), 7.30(\mathrm{~d}, J=$ $7.2 \mathrm{~Hz}, 1 \mathrm{H}), 7.40(\mathrm{t}, J=7.6 \mathrm{~Hz}, 2 \mathrm{H}), 7.66(\mathrm{~d}, J=9.2 \mathrm{~Hz}, 1 \mathrm{H})$, $7.85(\mathrm{~d}, J=7.2 \mathrm{~Hz}, 1 \mathrm{H}), 8.01(\mathrm{~d}, J=8.0 \mathrm{~Hz}, 1 \mathrm{H})$; ESI-MS: $\mathrm{m} / \mathrm{z} 286.5(\mathrm{M}+\mathrm{H})^{+}$. Elemental analysis $\mathrm{C}_{19} \mathrm{H}_{15} \mathrm{~N}_{3}$ calcd. (found) \%: C 79.97 (79.95), H 5.29 (5.26), N 14.72 (14.68).

2-(p-Tolyl)-N-phenylimidazo[1,2-a]pyridin-3-amine (6b) [28]: Yellow solid; Yield: 55 mg, $92 \%$; m.p.: $236-237{ }^{\circ} \mathrm{C}$; IR $\left(\mathrm{KBr}, v_{\max }, \mathrm{cm}^{-1}\right)$ : 3288 (-N-H str), 2922 (-C-H str), 1598 (-C=N str), 1434 (-C=C- str), 1180 (-C-N str); ${ }^{1} \mathrm{H}$ NMR (400 $\left.\mathrm{MHz}, \mathrm{CDCl}_{3}\right): \delta 7.89(\mathrm{~d}, J=8.2 \mathrm{~Hz}, 2 \mathrm{H}), 7.82(\mathrm{dd}, J=6.8$, $1.2 \mathrm{~Hz}, 1 \mathrm{H}), 7.63(\mathrm{dt}, J=9.1,1.1 \mathrm{~Hz}, 1 \mathrm{H}), 7.24-7.14(\mathrm{~m}$, $5 \mathrm{H}), 6.90-6.83(\mathrm{~m}, 1 \mathrm{H}), 6.75(\mathrm{td}, J=6.8,1.1 \mathrm{~Hz}, 1 \mathrm{H}), 6.62-$ 6.56 (m, 2H), 5.61 (s, 1H), 2.34 (s, 3 H); ESI-MS: m/z 300.1 $(\mathrm{M}+\mathrm{H})^{+}$. Elemental analysis $\mathrm{C}_{20} \mathrm{H}_{15} \mathrm{~N}_{3}$ calcd. (found) \%: $\mathrm{C} 80.23$ (80.19), H 5.71 (5.68), N 14.05 (14.01).

2-(4-Methoxyphenyl)-N-phenylimidazo[1,2-a]pyridin3-amine (6c) [30]: Off-white solid; Yield: $80 \%$; m.p.: 199$200{ }^{\circ} \mathrm{C}$; IR $\left(\mathrm{KBr}, v_{\max }, \mathrm{cm}^{-1}\right): 3278$ (-N-H str), 2918 (-C-H str), 1599 (-C=N str), 1438 (-C=C- str), 1182 (-C-N str); ${ }^{1} \mathrm{H}$ NMR $\left(400 \mathrm{MHz}, \mathrm{CDCl}_{3}\right): \delta 5.56(\mathrm{~s}, 1 \mathrm{H}), 6.55(\mathrm{~d}, J=8.4 \mathrm{~Hz}$, $2 \mathrm{H}), 6.66(\mathrm{t}, J=6.4 \mathrm{~Hz}, 1 \mathrm{H}), 6.82(\mathrm{t}, J=7.6 \mathrm{~Hz}, 1 \mathrm{H}), 7.06-$ $7.11(\mathrm{~m}, 2 \mathrm{H}), 7.22(\mathrm{~d}, J=7.2 \mathrm{~Hz}, 2 \mathrm{H}), 7.12(\mathrm{~d}, J=7.4 \mathrm{~Hz}$, 2H), $7.38(\mathrm{~d}, J=9.2 \mathrm{~Hz}, 1 \mathrm{H}), 7.48(\mathrm{~d}, J=7.2 \mathrm{~Hz}, 1 \mathrm{H}), 7.82$ $(\mathrm{d}, J=8.0 \mathrm{~Hz}, 1 \mathrm{H})$; ESI-MS: $m / z, 316.3(\mathrm{M}+\mathrm{H})^{+}$. Elemental analysis $\mathrm{C}_{20} \mathrm{H}_{15} \mathrm{~N}_{3} \mathrm{O}$ calcd. (found) \%: $\mathrm{C} 76.16$ (76.12), $\mathrm{H} 5.42$ (5.40), N 13.31 (13.29).

2-Cyclohexyl-N-phenylimidazo[1,2-a]pyridin-3-amine (6d) [31]: Pale yellow solid; Yield: $75 \%$; m.p.: $187-188{ }^{\circ} \mathrm{C}$; IR $\left(\mathrm{KBr}, v_{\max }, \mathrm{cm}^{-1}\right): 3205$ (-N-H str), 2925 (-C-H str), 1599 $\left(-\mathrm{C}=\mathrm{N}\right.$ str) 1494 (-C=C- str), 1170 (-C-N str); ${ }^{1} \mathrm{H}$ NMR (400 $\left.\mathrm{MHz}, \mathrm{CDCl}_{3}\right): \delta 1.20-1.30(\mathrm{~m}, 3 \mathrm{H}), 1.62-1.82(\mathrm{~m}, 8 \mathrm{H}), 5.30$ $(\mathrm{s}, 1 \mathrm{H}), 6.50(\mathrm{~d}, J=8.3 \mathrm{~Hz}, 2 \mathrm{H}), 6.71(\mathrm{t}, J=7.6 \mathrm{~Hz}, 1 \mathrm{H}), 6.82$ $(\mathrm{t}, J=7.4 \mathrm{~Hz}, 1 \mathrm{H}), 7.13-7.20(\mathrm{~m}, 3 \mathrm{H}), 7.58(\mathrm{~d}, J=8.8 \mathrm{~Hz}, 1 \mathrm{H})$, $7.77(\mathrm{~d}, J=6.6 \mathrm{~Hz}, 1 \mathrm{H})$; ESI-MS: $\mathrm{m} / z 292.5(\mathrm{M}+\mathrm{H})^{+}$. Elemental analysis $\mathrm{C}_{19} \mathrm{H}_{21} \mathrm{~N}_{3}$ calcd. (found) \%: $\mathrm{C} 78.34$ (78.32), H 7.28 (7.24), N 14.44 (14.42).

2-Ethyl-N-phenylimidazo[1,2-a]pyridin-3-amine (6e) [32]: White solid; Yield: $78 \%$; m.p.: $174-175{ }^{\circ} \mathrm{C}$; IR (KBr, $\left.v_{\max }, \mathrm{cm}^{-1}\right): 3218$ (-N-H str), 2929 (-C-H str), 1594 (-C=N str), 1487 (-C=C- str), 1168 (-C-N str); ${ }^{1} \mathrm{H}$ NMR $\left(400 \mathrm{MHz}, \mathrm{CDCl}_{3}\right)$ : $\delta 1.36(\mathrm{t}, J=6.8 \mathrm{~Hz}, 3 \mathrm{H}), 4.10(\mathrm{q}, J=6.8 \mathrm{~Hz}, 2 \mathrm{H}), 5.34(\mathrm{~s}, 1 \mathrm{H})$, $6.48(\mathrm{~d}, J=8.3 \mathrm{~Hz}, 2 \mathrm{H}), 6.71(\mathrm{t}, J=7.6 \mathrm{~Hz}, 1 \mathrm{H}), 6.82(\mathrm{t}, J=$ $7.4 \mathrm{~Hz}, 1 \mathrm{H}), 7.13-7.20(\mathrm{~m}, 2 \mathrm{H}), 7.56(\mathrm{~d}, J=7.8 \mathrm{~Hz}, 1 \mathrm{H}), 7.72$ $(\mathrm{d}, J=6.8 \mathrm{~Hz}, 1 \mathrm{H})$; ESI-MS: $\mathrm{m} / z 238.5(\mathrm{M}+\mathrm{H})^{+}$. Elemental analysis $\mathrm{C}_{15} \mathrm{H}_{15} \mathrm{~N}_{3}$ calcd. (found) \%: C 75.91 (75.89), H 6.36 (6.31), N 17.70 (17.69).

2-Isopropyl-N-phenylimidazo[1,2- $a$ ]pyridin-3-amine (6f): White solid; Yield: $80 \%$; m.p.: $216-217^{\circ} \mathrm{C}$; IR (KBr, $\left.v_{\max }, \mathrm{cm}^{-1}\right): 3200$ (-N-H str), 2921 (-C-H str), 1600 (-C=N str), 1495 (-C=C- str), 1154 (-C-N str); ${ }^{1} \mathrm{H}$ NMR $\left(400 \mathrm{MHz}, \mathrm{CDCl}_{3}\right)$ : $1.33(\mathrm{~d}, J=7.0 \mathrm{~Hz}, 6 \mathrm{H}), 3.10-3.20(\mathrm{~m}, 1 \mathrm{H}), 5.30(\mathrm{~s}, 1 \mathrm{H}), 6.50$ $(\mathrm{d}, J=7.4 \mathrm{~Hz}, 2 \mathrm{H}), 6.70(\mathrm{~d}, J=7.4 \mathrm{~Hz}, 1 \mathrm{H}), 6.82(\mathrm{t}, J=7.4$ $\mathrm{Hz}, 1 \mathrm{H}), 7.15-7.20(\mathrm{~m}, 3 \mathrm{H}), 7.60(\mathrm{~d}, J=8.7 \mathrm{~Hz}, 1 \mathrm{H}), 7.81(\mathrm{~d}$, $J=6.5 \mathrm{~Hz}, 1 \mathrm{H})$; ESI-MS: $m / z 252.5(\mathrm{M}+\mathrm{H})^{+}$. Elemental 
analysis $\mathrm{C}_{16} \mathrm{H}_{17} \mathrm{~N}_{3}$ calcd. (found) \%: C 76.45 (76.41), H 6.81 (6.79), N 16.71 (16.70).

N-Phenyl-2-(pyridin-2-yl)imidazo[1,2-a]pyridin-3amine (7a) [29]: Off-white solid; Yield: 84 \%; m.p.: 218-219 ${ }^{\circ} \mathrm{C}$; IR (KBr, $\left.v_{\max }, \mathrm{cm}^{-1}\right)$ : 3435 (-N-H str), 2929 (-C-H str), 1597 (-C=N str), 1494 (-C=C- str), 1148 (-C-N str); ${ }^{1} \mathrm{H}$ NMR $\left(400 \mathrm{MHz}, \mathrm{CDCl}_{3}\right): \delta 6.66(\mathrm{~d}, J=7.9 \mathrm{~Hz}, 2 \mathrm{H}), 6.76(\mathrm{t}, 7.6 \mathrm{~Hz}$, $1 \mathrm{H}), 6.92(\mathrm{t}, J=6.8 \mathrm{~Hz}, 1 \mathrm{H}), 7.12-7.25(\mathrm{~m}, 5 \mathrm{H}), 7.60-7.65$ (m, 2H), $7.77(\mathrm{t}, J=6.0 \mathrm{~Hz}, 1 \mathrm{H}), 8.19(\mathrm{~d}, J=7.9 \mathrm{~Hz}, 1 \mathrm{H}), 8.30$ (s, $1 \mathrm{H}, \mathrm{D}_{2} \mathrm{O}$ exchangeable), 8.56 (d, $\left.J=4.4 \mathrm{~Hz}, 1 \mathrm{H}\right)$; ESI-MS: $\mathrm{m} / z 287.5(\mathrm{M}+\mathrm{H})^{+}$. Elemental analysis $\mathrm{C}_{18} \mathrm{H}_{14} \mathrm{~N}_{4}$ calcd. (found) $\%$ : C 75.51 (75.50), H 4.95 (4.92), N 19.56 (19.54).

N-p-Tolyl-2-(pyridin-2-yl)imidazo[1,2-a]pyridin-3amine (7b): White solid; Yield: $80 \%$; m.p.: $168-169^{\circ} \mathrm{C}$; IR $\left(\mathrm{KBr}, \nu_{\max }, \mathrm{cm}^{-1}\right): 3440$ (-N-H str), 2922 (-C-H str), 1594 (-C=N str), 1493 (-C=C- str), 1151 (-C-N str); ${ }^{1} \mathrm{H}$ NMR $(400 \mathrm{MHz}$, $\left.\mathrm{CDCl}_{3}\right): \delta 2.36(\mathrm{~s}, 3 \mathrm{H}), 6.72(\mathrm{~d}, J=7.2 \mathrm{~Hz}, 2 \mathrm{H}), 6.54(\mathrm{~d}, J=$ $7.2 \mathrm{~Hz}, 2 \mathrm{H}), 6.70(\mathrm{t}, 7.6 \mathrm{~Hz}, 2 \mathrm{H}), 6.86(\mathrm{t}, J=6.8 \mathrm{~Hz}, 1 \mathrm{H})$, $7.64-7.66(\mathrm{~m}, 2 \mathrm{H}), 7.72(\mathrm{t}, J=6.0 \mathrm{~Hz}, 1 \mathrm{H}), 8.19(\mathrm{~d}, J=7.9$ $\mathrm{Hz}, 1 \mathrm{H}), 8.28\left(\mathrm{~s}, 1 \mathrm{H}, \mathrm{D}_{2} \mathrm{O}\right.$ exchangeable), $8.52(\mathrm{~d}, J=5.4 \mathrm{~Hz}$, $1 \mathrm{H})$; ESI-MS: $m / z 301.3(\mathrm{M}+\mathrm{H})^{+}$. Elemental analysis $\mathrm{C}_{19} \mathrm{H}_{16} \mathrm{~N}_{4}$ calcd. (found) \%: C 75.97 (75.94), H 5.36 (5.32), N 18.64 (18.62).

$\mathrm{N}$-(4-Methoxy-phenyl)-2-(pyridin-2-yl)imidazo[1,2a]pyridin-3-amine (7c): Brown solid; Yield: $82 \%$; m.p.: 183$185^{\circ} \mathrm{C}$; IR (KBr, $\left.v_{\max }, \mathrm{cm}^{-1}\right)$ : 3434 (-N-H str), 2928 (-C-H str), 1598 (-C=N str), 1496 (-C=C- str), 1156 (-C-N str); ${ }^{1} \mathrm{H}$ NMR (400 MHz, $\left.\mathrm{CDCl}_{3}\right): \delta 3.78(\mathrm{~s}, 3 \mathrm{H}), 6.68(\mathrm{~d}, J=6.8 \mathrm{~Hz}$, $2 \mathrm{H}), 6.82(\mathrm{~d}, J=7.0 \mathrm{~Hz}, 2 \mathrm{H}), 6.78(\mathrm{t}, 7.6 \mathrm{~Hz}, 2 \mathrm{H}), 6.90(\mathrm{t}, J=$ $6.8 \mathrm{~Hz}, 1 \mathrm{H}), 7.71-7.68(\mathrm{~m}, 2 \mathrm{H}), 7.76(\mathrm{t}, J=6.6 \mathrm{~Hz}, 1 \mathrm{H}), 8.22$ (d, $J=7.8 \mathrm{~Hz}, 1 \mathrm{H}), 8.31$ (s, $1 \mathrm{H}, \mathrm{D}_{2} \mathrm{O}$ exchangeable), 8.48 (d, $J=5.8 \mathrm{~Hz}, 1 \mathrm{H})$; ESI-MS: $\mathrm{m} / z 317.1(\mathrm{M}+\mathrm{H})^{+}$. Elemental analysis $\mathrm{C}_{16} \mathrm{H}_{16} \mathrm{~N}_{4} \mathrm{O}$ calcd. (found) \%: C 72.15 (72.13), H 5.12 (5.10), N 17.70 (17.67).

N-Cyclohexyl-2-(pyridin-2-yl)imidazo[1,2-a]pyridin3-amine (7d) [33]: Off-white solid; Yield: $84 \%$; m.p.: 113$114^{\circ} \mathrm{C}$; IR (KBr, $\left.v_{\max }, \mathrm{cm}^{-1}\right): 3265$ (-N-H str), 2928 (-C-H str), 1586 (-C=N str), 1476 (-C=C- str), 1147 (-C-N str); ${ }^{1} \mathrm{H}$ NMR $\left(400 \mathrm{MHz}, \mathrm{CDCl}_{3}\right): \delta 8.58(\mathrm{~s}, 1 \mathrm{H}), 8.18(\mathrm{~d}, J=8.4 \mathrm{~Hz}, 1 \mathrm{H})$, $7.96(\mathrm{~d}, J=6.8 \mathrm{~Hz}, 1 \mathrm{H}), 7.75(\mathrm{t}, J=6.4 \mathrm{~Hz}, 1 \mathrm{H}), 7.53(\mathrm{~d}, J=$ $9.2 \mathrm{~Hz}, 1 \mathrm{H}), 7.18-7.08(\mathrm{~m}, 2 \mathrm{H}), 6.76(\mathrm{~s}, 1 \mathrm{H}), 6.21$ (brs, $1 \mathrm{H}$, $\mathrm{D}_{2}$ Oexchangeble), 3.10 (brs, 1H), 1.98 (brs, 2H), 1.70 (brs, 2H), 1.54 (brs, 1H), 1.36-1.12 (m, 5H); ESI-MS: m/z 293.5 $(\mathrm{M}+\mathrm{H})^{+}$. Elemental analysis $\mathrm{C}_{18} \mathrm{H}_{20} \mathrm{~N}_{4}$ calcd. (found) \%: $\mathrm{C} 73.92$ (73.90), H 6.91 (6.89), N 19.14 (19.11).

N-Ethyl-2-(pyridin-2-yl)imidazo[1,2-a]pyridin-3amine (7e): Yellow oily liquid; Yield: $78 \%$; IR (KBr, $v_{\max }$, $\mathrm{cm}^{-1}$ ): 3268 (-N-H str), 2971 (-C-H str), 1597 (-C=N str), 1472 (-C=C- str), 1141 (-C-N str); ${ }^{1} \mathrm{H}$ NMR $\left(400 \mathrm{MHz}, \mathrm{CDCl}_{3}\right)$ : $\delta$ $1.38(\mathrm{t}, J=6.8 \mathrm{~Hz}, 3 \mathrm{H}), 4.14(\mathrm{q}, J=6.8 \mathrm{~Hz}, 2 \mathrm{H}), 6.08(\mathrm{~d}, J=$ $10.0 \mathrm{~Hz}, 1 \mathrm{H}), 6.71(\mathrm{t}, J=6.8 \mathrm{~Hz}, 1 \mathrm{H}), 7.04-7.13(\mathrm{~m}, 2 \mathrm{H})$, $7.50(\mathrm{~d}, J=9.2 \mathrm{~Hz}, 1 \mathrm{H}), 7.68-7.72(\mathrm{~m}, 1 \mathrm{H}), 7.95(\mathrm{~d}, J=7.0$ $\mathrm{Hz}, 1 \mathrm{H}), 8.14$ (d, $J=8.2 \mathrm{~Hz}, 1 \mathrm{H}), 8.52(\mathrm{~d}, J=4.8 \mathrm{~Hz}, 1 \mathrm{H})$; ESI-MS: $m / z 239.3(\mathrm{M}+\mathrm{H})^{+}$. Elemental analysis $\mathrm{C}_{14} \mathrm{H}_{14} \mathrm{~N}_{4}$ calcd. (found) \%: C 70.56 (70.51), H 5.93 (5.90), N 23.49 (23.47).

N-Isopropyl-2-(pyridin-2-yl)imidazo[1,2-a]pyridin-3amine (7f): Yellow syrupy liquid; Yield: $80 \%$; IR ( KBr, $v_{\max }$, $\mathrm{cm}^{-1}$ ): 3284 (-N-H str), 2964 (-C-H str), 1591 (-C=N str), 1476
(-C=C- str), 1136 (-C-N str); ${ }^{1} \mathrm{H}$ NMR $\left(400 \mathrm{MHz}, \mathrm{CDCl}_{3}\right): \delta$ $1.84(\mathrm{~d}, J=6.5 \mathrm{~Hz}, 6 \mathrm{H}), 3.40-3.48(\mathrm{~m}, 1 \mathrm{H}), 6.04(\mathrm{~d}, J=10.0$ $\mathrm{Hz}, 1 \mathrm{H}), 6.75(\mathrm{t}, J=6.8 \mathrm{~Hz}, 1 \mathrm{H}), 7.0-7.15(\mathrm{~m}, 2 \mathrm{H}), 7.52$ (d, $J$ $=9.2 \mathrm{~Hz}, 1 \mathrm{H}), 7.70-7.78(\mathrm{~m}, 1 \mathrm{H}), 7.99(\mathrm{~d}, J=7.0 \mathrm{~Hz}, 1 \mathrm{H})$, $8.17(\mathrm{~d}, J=8.2 \mathrm{~Hz}, 1 \mathrm{H}), 8.55(\mathrm{~d}, J=4.8 \mathrm{~Hz}, 1 \mathrm{H})$; ESI-MS: $\mathrm{m} / \mathrm{z} 253.5(\mathrm{M}+\mathrm{H})^{+}$. Elemental analysis $\mathrm{C}_{15} \mathrm{H}_{16} \mathrm{~N}_{4}$ calcd. (found) \%: C 71.41 (71.37), H 6.41 (6.39), N 22.19 (22.17).

\section{Experimental Procedure for antidiabetic studies [34-37]}

Acute toxicity: These studies were undertaken as per the standard experimental procedures. The $\mathrm{LD}_{50}$ cut-off value of the test compounds was fixed as $50 \mathrm{mg} / \mathrm{kg}$, as screening dose for evaluation of antidiabetic activity. All the animal experiments were conducted by the approval of Institutional Animal Ethics Committee, Anurag Group of Institutions (formerly Lalitha College of Pharmacy), Hyderabad, India. During the study period, guidelines of Committee for the Purpose of Control and Supervision of Experiments on Animals (CPCSEA), Institutional Animals Ethics Committee (IAEC) were followed for the maintenance of animals. The research work was approved by IAEC No: I/IAEC/LCP041/2014/SM40.

Alloxan induction of experimental diabetes: Overnightfasted Swiss albino mice are injected with alloxan $(50 \mathrm{mg} / \mathrm{kg})$ in saline buffer intraperitoneally and hyperglycemia was confirmed in animals after $72 \mathrm{~h}$. Blood glucose levels were determined and mice having blood glucose levels $<145 \mathrm{mg} / \mathrm{dl}$ were excluded from experiment and rest were divided into 10 groups.

Experimental design: Animals were divided into 14 groups of 6 animals $(n=6)$ : Group 1 diabetic animals (vehicle) received $0.5 \%$ CMC ( $1 \mathrm{~mL})$; Group 2 diabetic animals received insulin $50 \mathrm{mg} / \mathrm{kg}$. Groups (3-14) diabetic animals received compounds 6a-f/7a-f in a single dose of $50 \mathrm{mg} / \mathrm{kg}$ body weight per oral, respectively for 7 days continuously.

Blood glucose measurement: Blood was withdrawn from the tail vain each time. Blood glucose was measured at $0,3^{\text {rd }}$ and $7^{\text {th }}$ days interval. At the end of $0,3^{\text {rd }}$ and $7^{\text {th }}$ day, blood samples were withdrawn from a tail vein by snipping the tip of the tail and the blood glucose level was measured by Accu Sure Blood Glucose Monitoring System (Dr. Gene Health \& Wellness).

\section{RESULTS AND DISCUSSION}

$\mathrm{Xu}$ et al. [28] reported the synthesis of compounds $\mathbf{6 a}$, 6b using zinc iodide catalyzed conditions, Lacerda et al. [29] synthesized compound $\mathbf{6 c}, 7 \mathbf{a}$ using $\mathrm{AcOH}$ catalyzed reaction condition, Shinde et al. [31] reported the synthesis of compound 6d using $\mathrm{LaCl}_{3} \cdot 7 \mathrm{H}_{2} \mathrm{O}$. Kiselyov [32] reported the synthesis of compound 6e using N-fluoropyridinium salts, Dam et al. [33] reported the synthesis of $\mathbf{7 d}$ using Montmorillonite K-10 clay as catalyst. To the best of our knowledge, the synthesis of compounds $\mathbf{6 f}, \mathbf{7 b}, \mathbf{7 c}, \mathbf{7 e - 7 f}$ are new and have not been reported in the literature so far. Apart from the above mentioned reagents, in general imidazo[1,2-a]pyridines have been reported to be synthesized by different methodologies for this type of condensation reactions such as $\mathrm{Sc}(\mathrm{OTf})_{3}$ [38], $\mathrm{ZnCl}_{2}$ [39], $\mathrm{MgCl}_{2}$ [40], $\mathrm{ZrCl}_{4}$ [41], $\mathrm{SnCl}_{2} \cdot 2 \mathrm{H}_{2} \mathrm{O}$ [42], $\mathrm{HClO}_{4}$ [27], AcOH [28], p-TSA [43], montmorillonite K-10 [44], cellulose sulfuric acid [26], catalyst free and solvent free reaction condi- 


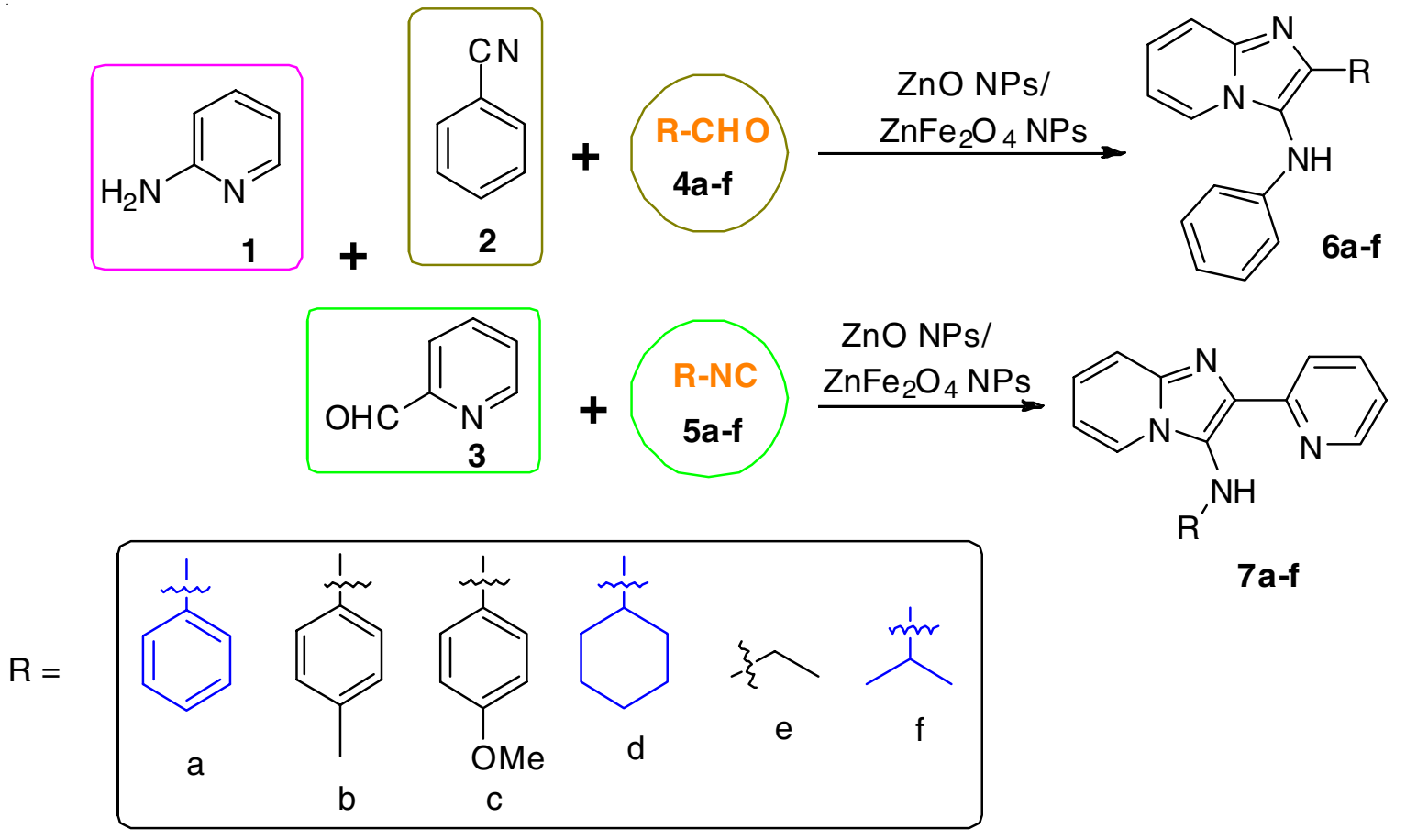

Scheme-I: Green synthesis of novel imidazo[1,2-a]pyridine derivative (6a-f/7a-f)

tions $[45,46]$. Most of these methods have many limitations in terms of lower product yields, toxicity, longer reaction time, higher energetic reaction conditions or use of hazardous and volatile organic solvents like acetonitrile, toluene, chloroform etc. Moreover, most of those catalysts are not reusable.

Our primary objective is to develop a green synthetic protocol using recyclable and reusable heterogeneous catalyst, green solvent and energy efficiency methods with benign reaction condition. The synthesis of final targets imidazo[1,2$a$ ]pyridines derivatives (6a-f and 7a-f) is illustrated in SchemeI. These compounds were synthesized via three component condensation reaction between aryl-alkyl-aldehydes (4a-f), 2aminopyridines and aryl-alkyl isocyanides (5a-f) utilizing reusable nanocrystalline $\mathrm{ZnO} / \mathrm{ZnFe}_{2} \mathrm{O}_{4}$ as catalyst in presence of green solvent 2-methyltetrahydrofuran and catalytic quantity of ethanol (Scheme-I) under microwave irradiation conditions.

The condensation reaction was successful (in presence of $\mathrm{ZnO}$ NPs) even without microwave irradiation but took longer reaction time (10-18 h) for the completion of the reaction [29]. In order to reduce the reaction time, attempted microwave conditions in presence of $\mathrm{ZnO} N P s / \mathrm{ZnFe}_{2} \mathrm{O}_{4} \mathrm{NPs}$ was successful by reducing the reaction time to $2-5 \mathrm{~min}$. The yields and quality of the products improved to a greater extent than the previously reported literature procedures. The salient features of our work lies in establishing the essential green principle ingredients such as usage of $\mathrm{ZnO} N P s / \mathrm{ZnFe}_{2} \mathrm{O}_{4}$ a reusable heterogeneous catalyst, 2-methyltetrahydrofuran, a green solvent and energy efficiency methods by utilizing microwave irradiation technique. The structural elucidation of the synthesized imidazo[1,2-a]pyridine derivatives was established by ${ }^{1} \mathrm{H}$ NMR, IR and mass spectroscopic techniques. As an example, the structural interpretation of 2-isopropyl-N-phenylimidazo[1,2-a]pyridin-3-amine (6f) is discussed here.

${ }^{1} \mathbf{H}$ NMR: The proton resonating at $7.81 \mathrm{ppm}(\mathrm{d}, 1 \mathrm{H})$,
$7.60 \mathrm{ppm}(\mathrm{d}, 1 \mathrm{H}), 7.15-7.20 \mathrm{ppm}(\mathrm{m}, 3 \mathrm{H}$, with overlap of phenyl proton) is assigned to the pyridine ring while the protons resonating at $6.50 \mathrm{ppm}(\mathrm{d}, 2 \mathrm{H}), 6.70 \mathrm{ppm}(\mathrm{d}, 1 \mathrm{H}), 6.82 \mathrm{ppm}(\mathrm{t}$, $1 \mathrm{H})$ and $7.15-7.20(\mathrm{~m}, 1 \mathrm{H})$ corresponds to the phenyl ring protons. The protons resonating in the aliphatic region 1.33 ppm $(\mathrm{d}, 6 \mathrm{H})$ and $3.10-3.20 \mathrm{ppm}(\mathrm{m}, 1 \mathrm{H})$ is assigned to the isopropyl group while the proton resonating at $5.30(\mathrm{~s}, 1 \mathrm{H}$, $\mathrm{D}_{2} \mathrm{O}$ exchangeable) is assigned to the $-\mathrm{NH}$ group, respectively.

Mass: The $m / z, 252.5$ with $(\mathrm{M}+\mathrm{H})^{+}$corresponds to the molecular weight of the desired compound i.e. compound $\mathbf{6 f}$.

IR: The stretching frequencies in the region 3200, 2921, $1600,1495,1154 \mathrm{~cm}^{-1}$ corresponds to $-\mathrm{NH},-\mathrm{CH},-\mathrm{C}=\mathrm{N},-\mathrm{C}-\mathrm{N}$ groups, respectively. The above analytical interpretation thus confirms the structure of the compound $6 \mathbf{f}$. Similarly, the structure of the remaining compounds in the series was characterized as described above.

Antidiabetic activity: Compilation of the antidiabetic activity results of the synthesized imidazo[1,2- $a$ ]pyridine derivatives (6a-f/7a-f) is tabulated in Table-1. All these derivatives exhibited significant hypoglycemic property, but with a certain degree of variation. A major increase in blood glucose was determined in diabetic rats. All the compounds 6a-f/7a-f had shown a significant reduction in blood glucose as compared to control diabetic rats at $50 \mathrm{mg} / \mathrm{kg}$ body weight for $3^{\text {rd }}$ and $7^{\text {th }}$ days.

Insulin was used as a standard drug for the purpose of the study and showed $68.88 \%$ blood glucose lowering activity at the dose of $50 \mathrm{mg} / \mathrm{kg}$.p.o. From Table-1, it is observed that 7a-f imidazo[1,2- $a$ ]pyridine series of compounds exhibited significant hypoglycemic activity when compare to 6a-f series of imidazo[1,2-a]pyridine. Compounds 7d (67.0\%), 7e (65.54 $\%)$ and $7 f(64.64 \%)$ with substitution $\mathrm{R}=$ cyclohexyl, ethyl and isopropyl showed nearly equivalent hypoglycemic activity similar to the standard drug insulin $(50 \mathrm{mg} / \mathrm{kg} \mathrm{b.w})$ in reducing the blood glucose level, while the compounds $7 \mathbf{a}(58.06 \%)$, 
TABLE-1

RESULTS OF HYPOGLYCEMIC EFFECTS OF HYDRAZONE DERIVATIVES (6a-f/7a-f)

\begin{tabular}{|c|c|c|c|c|}
\hline \multirow{2}{*}{ Treatment (mg/kg b.w p.o) } & \multicolumn{4}{|c|}{ Blood glucose level (mg/dl) } \\
\hline & Day-0 & Day-3 & Day-7 & Hyperglycemic activity (\%) \\
\hline Control $(0.5 \%$ CMC $)$ & $345.0 \pm 2.48$ & $376.30 \pm 3.54 * *$ & $395.1 \pm 3.03 * *$ & - \\
\hline Insulin & $354.0 \pm 1.96$ & $146.30 \pm 2.74 * *$ & $110.14 \pm 2.46^{* *}$ & 68.88 \\
\hline $6 \mathbf{a}$ & $346.5 \pm 2.46$ & $262.10 \pm 2.26^{* *}$ & $157.40 \pm 3.46^{* *}$ & 54.57 \\
\hline 6b & $343.0 \pm 3.25$ & $255.10 \pm 2.72 * *$ & $154.64 \pm 2.46^{* *}$ & 55.02 \\
\hline $6 c$ & $353.4 \pm 2.27$ & $282.3 \pm 3.65^{* *}$ & $159.20 \pm 1.69 * *$ & 54.95 \\
\hline 6d & $352.6 \pm 2.26$ & $280.20 \pm 3.80 * *$ & $154.42 \pm 1.92 * *$ & 56.20 \\
\hline $6 e$ & $354.3 \pm 2.46$ & $246.50 \pm 3.13^{* *}$ & $157.30 \pm 1.46^{* *}$ & 55.64 \\
\hline 6f & $342.4 \pm 1.88$ & $292.30 \pm 3.06^{* *}$ & $154.30 \pm 1.46^{* *}$ & 54.93 \\
\hline $7 \mathbf{a}$ & $357.3 \pm 2.37$ & $262.35 \pm 1.68 * *$ & $149.84 \pm 3.01 * *$ & 58.06 \\
\hline $7 b$ & $325.8 \pm 2.25$ & $207.21 \pm 3.13^{* *}$ & $120.42 \pm 2.19 * *$ & 63.03 \\
\hline $7 c$ & $334.6 \pm 2.05$ & $183.50 \pm 1.94 * *$ & $125.30 \pm 2.66^{* *}$ & 62.55 \\
\hline $7 d$ & $348.5 \pm 2.86$ & $239.44 \pm 2.16^{* *}$ & $114.72 \pm 1.98 * *$ & 67.08 \\
\hline $7 e$ & $338.6 \pm 2.40$ & $225.28 \pm 2.46^{* *}$ & $117.35 \pm 1.60 * *$ & 65.54 \\
\hline $7 f$ & $339.3 \pm 3.39$ & $239.22 \pm 2.13 * *$ & $116.50 \pm 3.99 * *$ & 64.64 \\
\hline
\end{tabular}

Values are represented as mean \pm SEM. Data were analyzed using analysis of variance and group means were compared with Tukey-Kramer Post ANOVA test. The values were considered when $\mathrm{P}<0.01$. **P $<0.001$; Tabulated data are expressed as mean \pm SEM; $(n=6)$.

7b $(63.03 \%)$ and $7 \mathbf{c}(62.55 \%)$ with substitution $\mathrm{R}=$ phenyl, para-methoxy phenyl, $p$-methyl-phenyl showed considerably good hypoglycemic activity. In case of imidazo[1,2-a]pyridine series, all the compounds viz., compounds $\mathbf{6 a}(54.57 \%), \mathbf{6 b}$ $(55.02 \%), \mathbf{6 c}(54.95 \%), \mathbf{6 d}(56.20 \%), \mathbf{6 e}(\mathrm{R}=55.64 \%)$ and 6f $(54.93 \%)$ displayed moderate hypoglycemic activity.

In general from the determined results, it is concluded that in the main scaffold of imidazo[1,2- $a$ ]pyridine nucleus, inclusion of pyridine ring in the second position and $\mathrm{N}$-substituted aryl/ alkyl moieties viz., compounds (7a-f) reduced the glucose level in diabetic rats. However, the impact of $\mathbf{7 d}, \mathbf{7 e}$ and $\mathbf{7 f}$ is more pronounced in alloxan induced diabetic rats.

Further exploration of the above established results towards the synthesis, toxicity studies and mode of action of hypoglycemic activity may lead to the development of an efficient drug candidate for diabetes mellitus.

\section{Conclusion}

The present paper illustrates the green synthesis of imidazo[1,2-a]pyridine derivatives (6a-f/7a-f) using the green parameters such as (i) $\mathrm{ZnO}$ nanoparticle/ $\mathrm{ZnFe}_{2} \mathrm{O}_{4}$ (a magnetically separable heterogeneous catalyst), (ii) 2-methyltetrahydrofuran (green solvent) and (iii) microwave irradiation (energy efficiency reducing time period of the reaction) utilizing the raw materials viz., such as aryl/alkyl-aldehydes, 2-aminopyridine and aryl/ alkyl isocyanides. Evaluation of antidiabetic activity of the synthesized imidazo $[1,2-a]$ pyridine derivatives $\mathbf{6 a - f} / 7 \mathbf{a}-\mathbf{f}$ revealed that most of the compounds exhibited significant hypoglycemic property when compare to insulin as the reference drug. In general, it is concluded that in the main scaffold of imidazo$[1,2-a]$ pyridine nucleus, inclusion of pyridine ring in the second position and $\mathrm{N}$-substituted aryl/alkyl moieties such as compounds $7 \mathbf{d}(67.0 \%), 7 \mathbf{e}(65.54 \%)$ and $7 \mathbf{f}(64.64 \%)$ with substitution $\mathrm{R}=$ cyclohexyl, ethyl and isopropyl reduced the glucose level in diabetic rats.

\section{ACKNOWLEDGEMENTS}

The authors are thankful to Advanced Analytical Lab, Andhra University, Visakhapatnam, India for conducting the ${ }^{1} \mathrm{H}$ NMR and Mass spectral analysis.

\section{REFERENCES}

1. K. Gudmundsson and S.D. Boggs, PCT Int. Appl., WO 2006026703, CAN, 274, 144 (2006).

2. K.C. Rupert, J.R. Henry, J.H. Dodd, S.A. Wadsworth, D.E. Cavender, G.C. Olini, B. Fahmy and J. Siekierka, Bioorg. Med. Chem. Lett., 13, 347 (2003);

https://doi.org/10.1016/S0960-894X(02)01020-X.

3. A.T. Baviskar, C. Madaan, R. Preet, P. Mohapatra, V. Jain, A. Agarwal, S.K. Guchhait, C.N. Kundu, U.C. Banerjee and P.V. Bharatam, J. Med. Chem., 54, 5013 (2011); https://doi.org/10.1021/jm200235u.

4. N.M. Shukla, D.B. Salunke, E. Yoo, C.A. Mutz, R. Balakrishna and S.A. David, Bioorg. Med. Chem., 20, 5850 (2012); https://doi.org/10.1016/j.bmc.2012.07.052.

5. G.C. Moraski, L.D. Markley, P.A. Hipskind, H. Boshoff, S. Cho, S.G. Franzblau and M.J. Miller, ACS Med. Chem. Lett., 2, 466 (2011); https://doi.org/10.1021/ml200036r.

6. T. Ueda, K. Mizushige, K. Yukiiri, T. Takahashi and M. Kohno, Cerebrovasc. Dis., 16, 396 (2003); https://doi.org/10.1159/000072563.

7. K. Mizushige, T. Ueda, K. Yukiiri and H. Suzuki, Cardiovasc. Drug Rev, 20, 163 (2002);

https://doi.org/10.1111/j.1527-3466.2002.tb00085.x.

8. T. Swainston Harrison and G.M. Keating, CNS Drugs, 19, 65 (2005); https://doi.org/10.2165/00023210-200519010-00008.

9. R.F. Holcombe, C.E. McLaren and T. Milovanovic, Cancer Detect. Prev., 30, 94 (2006);

https://doi.org/10.1016/j.cdp.2005.08.002.

10. E.C. Mun, J.M. Mayol, M. Riegler, T.C. O'Brien, O.C. Farokhzad, J.C. Song, C. Pothoulakis, B.J. Hrnjez and J.B. Matthews, Gastroenterology, 114, 1257 (1998); https://doi.org/10.1016/S0016-5085(98)70432-9.

11. S.Z. Langer, S. Arbilla, J. Benavides and B. Scatton, Adv. Biochem. Psychopharmacol., 46, 61 (1990).

12. A. Berson, V. Descatoire, A. Sutton, D. Fau, B. Maulny, N. Vadrot, G. Feldmann, B. Berthon, T. Tordjmann and D. Pessayre, J. Pharmacol. Exp. Ther, 299, 793 (2001).

13. B. Saletu, J. GrNberger and L. Linzmayer, Int. Clin. Psychopharmacol., 1, 145 (1986); https://doi.org/10.1097/00004850-198604000-00006.

14. J.J. Kaminski, B. Wallmark, C. Briving and B.M. Andersson, J. Med. Chem., 34, 533 (1991); https://doi.org/10.1021/jm00106a008

15. Y. Jin, M.C. Rho, K. Gajulapati, H.Y. Jung, S.K. Boovanahalli, J.H. Lee, G.-Y. Song, J.H. Choi, Y.K. Kim, K. Lee and Y. Choi, Bull. Korean Chem. Soc., 30, 1297 (2009); https://doi.org/10.5012/bkcs.2009.30.6.1297. 
16. M.H. Wiegand, Drugs, 68, 2411 (2008); https://doi.org/10.2165/0003495-200868170-00001.

17. K.S. Gudmundsson, J.D. Williams, J.C. Drach and L.B. Townsend, J. Med. Chem., 46, 1449 (2003); https://doi.org/10.1021/jm020339r.

18. J.J. Kaminski and A.M. Doweyko, J. Med. Chem., 40, 427 (1999); https://doi.org/10.1021/jm950700s.

19. R.J. Boerner and H.J. Moller, Psychopharmakotherapie, 4, 145 (1997).

20. D.J. Sanger, Behav. Pharmacol., 6, 116 (1995).

21. T.H. Al-Tel and R.A. Al-Qawasmeh, Eur. J. Med. Chem., 45, 5848 (2010); https://doi.org/10.1016/j.ejmech.2010.09.049.

22. J.E. Starrett, T.A. Montzka, A.R. Crosswell and R.L. Cavanagh, J. Med. Chem., 32, 2204 (1989); https://doi.org/10.1021/jm00129a028.

23. B. Mirmashhori, N. Azizi and M.R. Saidi, J. Mol. Catal. Chem., 247, 159 (2006); https://doi.org/10.1016/j.molcata.2005.11.042.

24. A. Gueiffier, S. Mavel, M. Lhassani, A. Elhakmaoui, R. Snoeck, G. Andrei, O. Chavignon, J.C. Teulade, M. Witvrouw, J. Balzarini, E. De Clercq and J.-P. Chapat, J. Med. Chem., 41, 5108 (1998); https://doi.org/10.1021/jm981051y.

25. K. Groebke, L. Weber and F. Mehlin, Synlett, 661 (1998); https://doi.org/10.1055/s-1998-1721.

26. H. Bienayme and K. Bouzid, Angew. Chem. Int. Ed., 37, 2234 (1998); https://doi.org/10.1002/(SICI)1521-3773(19980904)37:16<2234:: AID-ANIE2234>3.0.CO;2-R.

27. C. Blackburn, B. Guan, P. Fleming, K. Shiosaki and S. Tsai, Tetrahedron Lett., 39, 3635 (1998); https://doi.org/10.1016/S0040-4039(98)00653-4.

28. H. Xu, C. Ma, Z. Wu and G. Haung, Synthesis, 48, 351 (2016); https://doi.org/10.1055/s-0035-1560375.

29. R.B. Lacerda, C.K. de Lima, L.L. da Silva, N.C. Romeiro, A.L. Miranda, E.J. Barreiro and C.A. Fraga, Bioorg. Med. Chem., 17, 74 (2009); https://doi.org/10.1016/j.bmc.2008.11.018.

30. L.B.E. De Jesus De, F. Carlos, A. Manssour, S.G. Zapata, S. Roberto and T.R. Menegatti, Pharmaceutical Compositions containing 1-Methyl3,6,7,8-Tetrahydropirazolo[3,4-b]pyrrolo[4,3-d]Pyridine-6,8-dione Derivatives, Use and Process for Preparing Them, WO2006092032A2 (2006).

31. H.A. Shinde, M. Srilaxmi, B. Satpathi and D.S. Sharada, Tetrahedron Lett., 55, 5915 (2014); https://doi.org/10.1016/j.tetlet.2014.08.126.
32. A.S. Kiselyov, Tetrahedron Lett., 46, 4487 (2005); https://doi.org/10.1016/j.tetlet.2005.04.124.

33. J. Dam, Z. Ismail, T. Kurebwa, N. Gangat, L. Harmse, H.M. Marques, A. Lemmerer, M.L. Bode and C.B. de Koning, Eur. J. Med. Chem., 126, 353 (2017); https://doi.org/10.1016/j.ejmech.2016.10.041.

34. J.T. Litchfield and E.J.A. Wilcoxon, J. Pharmacol. Exp. Ther, 96, 99 (1949).

35. P.V. Raghavan, Expert Consultant, CPCSEA, OECD, Guideline No. 420 (2000).

36. R.J. Jarrett, H. Keen and C. Hardwick, Diabetes, 19, 724 (1970); https://doi.org/10.2337/diab.19.10.724.

37. C.R. Mackerer, R.N. Saunders, J.R. Haettinger and M.A. Mehlman, J. Toxicol. Environ. Health, 2, 139 (1976); https://doi.org/10.1080/15287397609529422.

38. A. Shaabani, A. Maleki, J. Moghimi Rad and E. Soleimani, Chem. Pharm. Bull. (Tokyo), 55, 957 (2007); https://doi.org/10.1248/cpb.55.957.

39. A.L. Rousseau, P. Matlaba and C.J. Parkinson, Tetrahedron Lett., 48, 4079 (2007); https://doi.org/10.1016/j.tetlet.2007.04.008.

40. L.R. Odell, M. Nilsson, J. Gising, O. Lagerlund, D. Muthas, A. Nordqvist, A. Karlen and M. Larhed, Bioorg. Med. Chem. Lett., 19, 4790 (2009); https://doi.org/10.1016/j.bmcl.2009.06.045.

41. S. Guchhait and C. Madaan, Synlett, 628 (2009); https://doi.org/10.1055/s-0028-1087915.

42. A. Shaabani, E. Soleimani, A. Sarvary, H. Rezayan and A. Maleki, Chin. J. Chem., 27, 369 (2009); https://doi.org/10.1002/cjoc. 200990060.

43. A. Shaabani, E. Soleimani, A. Maleki and J. Moghimi-Rad, Synth. Commun., 38, 1090 (2008); https://doi.org/10.1080/00397910701862931.

44. R.S. Varma and D. Kumar, Tetrahedron Lett., 40, 7665 (1999); https://doi.org/10.1016/S0040-4039(99)01585-3.

45. M. Adib, M. Mahdavi, M.A. Noghani and P. Mirzaei, Tetrahedron Lett., 48, 7263 (2007); https://doi.org/10.1016/j.tetlet.2007.08.049.

46. S. Vidyacharan, A.H. Shinde, B. Satpathi and D.S. Sharada, Green Chem., 16, 1168 (2014); https://doi.org/10.1039/c3gc42130a. 